\title{
Further acceleration of the Simpson method for solving nonlinear equations
}

\author{
R. Thukral
}

Padé Research Centre, 39 Deanswood Hill, Leeds, West Yorkshire, LS17 5JS, England

\begin{abstract}
There are two aims of this paper, firstly, we present an improvement of the classical Simpson third-order method for finding zeros a nonlinear equation and secondly, we introduce a new formula for approximating second-order derivative. The new Simpson-type method is shown to converge of the order four. Per iteration the new method requires same amount of evaluations of the function and therefore the new method has an efficiency index better than the classical Simpson method. We examine the effectiveness of the new fourthorder Simpson-type method by approximating the simple root of a given nonlinear equation. Numerical comparisons are made with classical Simpson method to show the performance of the presented method.
\end{abstract}

Keywords: Simpson method, Newton method, Simple root, Nonlinear equation, Root-finding, Order of convergence.

Subject Classifications: AMS (MOS): 65H05, 41A25.

Date of Publication: 30.5.2018

DOI: $10.24297 / j a m . v 14 i 2.7415$

ISSN: 2347-1921

Volume: 14 Issue: 02

Journal: Journal of Advances in Mathematics

Website: https://cirworld.com

This work is licensed under a Creative Commons Attribution 4.0 International License. 


\section{Introduction}

In this paper, we present a new fourth-order iterative method to find a simple root of the nonlinear equation. It is well known that the techniques to solve nonlinear equations have many applications in science and engineering. We shall utilise two well-known techniques, namely the classical Simpson method and the classical Newton method, for their simplicity with convergence order of three and two respectively $[3,4,5]$. The new Simpson-type method requires same amount of evaluations of the function as the classical Simpson method. We shall prove that the new Simpson-type iterative method has a better efficiency index than the classical Simpson method [1,2,4-7]. The prime motive for the development of the new Simpson-type method was to increase the order of convergence of classical Simpson method. Furthermore, we introduce a new formula for approximating the second-order derivative of a function.

\section{Development of the method and analysis of convergence}

Let $f(x)$ be a real function with a simple root $\alpha$ and let $\left\{x_{n}\right\}_{n \in \square}$ be a sequence of real numbers that converge towards $\alpha$. The order of convergence $p$ is given by

$$
\lim _{n \rightarrow \infty} \frac{x_{n+1}-\alpha}{\left(x_{n}-\alpha\right)^{p}}=\omega \neq 0
$$

where $p \in \mathfrak{R}^{+}$and $\omega$ is the asymptotic error constant. Also, let $e_{k}=x_{k}-\alpha$ be the error in the $k$ th iteration, then the relation

$e_{k+1}=\zeta e_{k}^{p}+\mathrm{O}\left(e_{k}^{p+1}\right)$

is the error equation. If the error equation exists, then $p$ is the order of convergence of the iterative method $[3,7,8,10]$.

Furthermore, the efficiency of the method is measured by the concept of efficiency index, given by

$$
E I(r, p)=\sqrt[r]{p}
$$

where $r$ is the number of function evaluations of the method and $p$ is the order of the convergence of the method [7]. In order to approximate the order of convergence of the method we use the following formula. Suppose that $x_{n-1}, x_{n}$ and $x_{n+1}$ are three successive iterations closer to the root $\alpha$ of (1). Then the computational order of convergence may be approximated by

$\mathrm{COC} \approx \frac{\ln \left|\lambda_{n} \div \lambda_{n-1}\right|}{\ln \left|\lambda_{n-1} \div \lambda_{n-2}\right|}$

where $\lambda_{i}=f\left(x_{i}\right) \div f^{\prime}\left(x_{i}\right)$, [9].

In order to construct the new fourth-order iterative method, we state the essentials of the established thirdorder Simpson method.

\subsection{The Classical Simpson third-order method}

Since this method is well established, we shall state the essential expressions used in order to calculate the root of the given nonlinear equation. Hence the Simpson third-order method is given as 


$$
x_{n+1}=x_{n}-\frac{6 f\left(x_{n}\right)}{f^{\prime}\left(x_{n}\right)+4 f^{\prime}\left(z_{n}\right)+f^{\prime}\left(y_{n}\right)},
$$

where

$$
u_{n}=\frac{f\left(x_{n}\right)}{f^{\prime}\left(x_{n}\right)},
$$

$y_{n}=x_{n}-u_{n}$,

$z_{n}=x_{n}-2^{-1} u_{n}$

$x_{0}$ is the initial approximation and provided that the denominator of (5) is not equal to zero.

\subsection{The Simpson-type fourth-order method}

In this section we present a new scheme to find simple root of a nonlinear equation. In order to increase the order of convergence of the classical Simpson method, we shall introduce a new factor in (5). Hence, the general formula of the new fourth-order Simpson method is given by

$$
x_{n+1}^{(i)}=x_{n}-\frac{6 f\left(x_{n}\right)}{f^{\prime}\left(x_{n}\right)+4 f^{\prime}\left(z_{n}\right)+f^{\prime}\left(y_{n}\right)}-2^{-2}\left(\frac{\delta_{i}}{f^{\prime}\left(x_{n}\right)}\right)^{2} u_{n}^{3},
$$

where

$$
\begin{aligned}
& \delta_{1}=\frac{2\left(f^{\prime}\left(y_{n}\right)-f^{\prime}\left(z_{n}\right)\right)}{u_{n}}, \\
& \delta_{2}=\frac{2\left(f^{\prime}\left(x_{n}\right)-f^{\prime}\left(z_{n}\right)\right)}{u_{n}},
\end{aligned}
$$

$\delta_{3}=\frac{5 f^{\prime}\left(x_{n}\right)-4 f^{\prime}\left(z_{n}\right)-f^{\prime}\left(y_{n}\right)}{3 u_{n}}$,

$\delta_{4}=\frac{4 f^{\prime}\left(z_{n}\right)-f^{\prime}\left(y_{n}\right)-3 f^{\prime}\left(x_{n}\right)}{(m+1) w_{n}+m v_{n}-2 m u_{n}}$,

$v_{n}=\frac{f\left(x_{n}\right)}{f^{\prime}\left(y_{n}\right)}$,

$w_{n}=\frac{f\left(x_{n}\right)}{f^{\prime}\left(z_{n}\right)}$, 


\section{Theorem 1}

Assume that the function $f: I \subset \mathfrak{R} \rightarrow \mathfrak{R}$ for an open interval I has a simple root $\alpha \in I$. Let $f(x)$ be sufficiently smooth in the interval $l$, then the order of convergence of the new method defined by (9) is four.

\section{Proof}

Let $\alpha$ be a simple root of $f(x)$, i.e. $f(\alpha)=0$ and $f^{\prime}(\alpha) \neq 0$, and the error is expressed as

$e=x-\alpha$.

Using Taylor expansion, we have

$f\left(x_{n}\right)=f(\alpha)+f^{\prime}(\alpha) e_{n}+2^{-1} f^{\prime \prime}(\alpha) e_{n}^{2}+\cdots$.

Taking $f(\alpha)=0$ and simplifying, expression (17) becomes

$f\left(x_{n}\right)=f^{\prime}(\alpha)\left[e_{n}+c_{2} e_{n}^{2}+c_{3} e_{n}^{3}+\cdots\right]$.

where

$c_{k}=\frac{f^{(k)}(\alpha)}{(k !) f^{\prime}(\alpha)}$ for $k=2,3,4, \ldots$

Furthermore, we have

$f^{\prime}\left(x_{n}\right)=f^{\prime}(\alpha)\left[1+2 c_{2} e_{n}+3 c_{3} e_{n}^{2}+\cdots\right]$,

$f^{\prime \prime}\left(x_{n}\right)=f^{\prime}(\alpha)\left[2 c_{2}+6 c_{3} e_{n}+\cdots\right]$.

Dividing (18) by (20), we get

$\frac{f\left(x_{n}\right)}{f^{\prime}\left(x_{n}\right)}=e_{n}-c_{2} e_{n}^{2}+2\left(c_{2}^{2}-c_{3}\right) e_{n}^{3}+\cdots$

and hence, we have

$y_{n}=e_{n}-\frac{f\left(x_{n}\right)}{f^{\prime}\left(x_{n}\right)}=c_{2} e_{n}^{2}-2\left(c_{2}^{2}-c_{3}\right) e_{n}^{3}-\cdots$

The expansion of $f^{\prime}\left(y_{n}\right)$ about $\alpha$ is given as

$f^{\prime}\left(y_{n}\right)=f^{\prime}(\alpha)\left[c_{2} e_{n}^{2}-2\left(c_{2}^{2}-c_{3}\right) e_{n}^{3}-\cdots\right]$

Since from (10) we have 
$z_{n}=x_{n}-2^{-1}\left(\frac{f\left(x_{n}\right)}{f^{\prime}\left(x_{n}\right)}\right)=\frac{e_{n}}{2}+\frac{c_{2} e_{n}^{2}}{2}+\cdots$

Taylor expansion of $f^{\prime}\left(z_{n}\right)$ about $\alpha$ is

$f^{\prime}\left(z_{n}\right)=f^{\prime}(\alpha)\left[c_{2} e_{n}^{2}-2\left(c_{2}^{2}-c_{3}\right) e_{n}^{3}-\cdots\right]$

Furthermore, expansion of $v_{n}, w_{n}$ is given by

$v_{n}=\frac{f\left(x_{n}\right)}{f^{\prime}\left(y_{n}\right)}=e_{n}+c_{2} e_{n}^{2}+\cdots$,

$w_{n}=\frac{f\left(x_{n}\right)}{f^{\prime}\left(z_{n}\right)}=2^{-1} e_{n}+2^{-1} c_{2} e_{n}^{2}+\cdots$,

Approximations of second-order derivative are given as

$\delta_{1}=\frac{2\left(f^{\prime}\left(y_{n}\right)-f^{\prime}\left(z_{n}\right)\right)}{u_{n}}=2 c_{2}+\left(\frac{3}{2}\right) c_{3} e_{n}+\cdots$,

$\delta_{2}=\frac{2\left(f^{\prime}\left(x_{n}\right)-f^{\prime}\left(z_{n}\right)\right)}{u_{n}}=2 c_{2}+\left(\frac{9}{2}\right) c_{3} e_{n}+\cdots$,

$\delta_{3}=\frac{5 f^{\prime}\left(x_{n}\right)-4 f^{\prime}\left(z_{n}\right)-f^{\prime}\left(y_{n}\right)}{3 u_{n}}=2 c_{2}+4 c_{3} e_{n}+\cdots$,

$\delta_{4}=\frac{4 f^{\prime}\left(z_{n}\right)-f^{\prime}\left(y_{n}\right)-3 f^{\prime}\left(x_{n}\right)}{(m-1) w_{n}+m v_{n}-2 m u_{n}}=2 c_{2}+6 c_{3} e_{n}+\cdots$,

The denominator of (5) is given as

$f^{\prime}\left(x_{n}\right)+4 f^{\prime}\left(z_{n}\right)+f^{\prime}\left(y_{n}\right)=6+6 c_{2} e_{n}+6\left(c_{2}^{2}+c_{3}\right) e_{n}^{2}+\cdots$

Dividing the numerator by denominator of (5), we get

$$
e_{n}-c_{2}^{2} e_{n}^{3}+3\left(c_{2}^{3}-c_{2} c_{3}\right) e_{n}^{4}+\cdots
$$

It is well established that the error equation of (5) is

$e_{n+1}=c_{2}^{2} e_{n}^{3}+\cdots$

The improvement factors introduce in (9) are

$2^{-2}\left(\frac{\delta_{1}}{f^{\prime}\left(x_{n}\right)}\right)^{2} u_{n}^{3}=c_{2}^{2} e_{n}^{3}+\left(3 c_{2} c_{3}-7 c_{2}^{3}\right) e_{n}^{4}+\cdots$ 


$$
\begin{aligned}
& 2^{-2}\left(\frac{\delta_{2}}{f^{\prime}\left(x_{n}\right)}\right)^{2} u_{n}^{3}=c_{2}^{2} e_{n}^{3}+\left(\left(\frac{9}{2}\right) c_{2} c_{3}-7 c_{2}^{3}\right) e_{n}^{4}+\cdots, \\
& 2^{-2}\left(\frac{\delta_{3}}{f^{\prime}\left(x_{n}\right)}\right)^{2} u_{n}^{3}=c_{2}^{2} e_{n}^{3}+\left(4 c_{2} c_{3}-7 c_{2}^{3}\right) e_{n}^{4}+\cdots, \\
& 2^{-2}\left(\frac{\delta_{4}}{f^{\prime}\left(x_{n}\right)}\right)^{2} u_{n}^{3}=c_{2}^{2} e_{n}^{3}+\left(6 c_{2} c_{3}-7 c_{2}^{3}\right) e_{n}^{4}+\cdots
\end{aligned}
$$

Substituting appropriate expressions in (9) and we shall obtain the error equations for the new iterative methods defined by (9). Using the four improvement factor we have obtained four different fourth-order methods, hence the error equation for these methods are given as

$e_{n+1}^{(1)}=\left(4 c_{2}^{3}+\left(\frac{3}{2}\right) c_{2} c_{3}\right) e_{n}^{4}+\mathrm{O}\left[e_{n}^{5}\right]$,

$e_{n+1}^{(2)}=\left(4 c_{2}^{3}-\left(\frac{3}{2}\right) c_{2} c_{3}\right) e_{n}^{4}+\mathrm{O}\left[e_{n}^{5}\right]$

$e_{n+1}^{(3)}=\left(4 c_{2}^{3}-c_{2} c_{3}\right) e_{n}^{4}+\mathrm{O}\left[e_{n}^{5}\right]$

$e_{n+1}^{(4)}=\left(4 c_{2}^{3}-3 c_{2} c_{3}\right) e_{n}^{4}+\mathrm{O}\left[e_{n}^{5}\right]$

respectively. The error equation (40)-(43) establishes the fourth-order convergence of the new Simpson-type method defined by (9).

\section{Numerical examples}

The new fourth-order methods given by (9) is employed to solve nonlinear equations with simple root. To demonstrate the performance of the new Simpson-type fourth-order method, ten particular nonlinear equations are used. The estimates are given of the approximate solutions produced by the methods considered and list the errors obtained by each of the methods. To determine the efficiency index of the new method, formula (3) will be used. Hence, the efficiency index of the new iterative method given by (9) is $\sqrt[4]{4} \approx 1.4142$. the efficiency index of the classical Simpson third-order method is given by (5) is $\sqrt[4]{3} \approx 1.3161$. It is shown that the efficiency index of the new fourth-order method is much better than the classical Simpson third-order method. Ten particular test functions are displayed in table 1 . The difference between the simple root $\alpha$ and the approximation $x_{n}$ for test functions with initial guess $x_{0}$ are displayed in table 2. In fact, $x_{n}$ is calculated by using the same total number of function evaluations for all methods. Furthermore, we display the computational order of convergence approximations in table 3. From the tables we observe that the $\mathrm{COC}$ perfectly coincides with the theoretical result.

Table 1 Test functions and their simple roots.

\begin{tabular}{|l|l|l|l|}
\hline Functions & Parameter & Simple Root & Initial guess \\
\hline$f_{1}(x)=\ln \left(1+x^{2}\right)+\exp (x) \sin (x)$ & $m=-4$ & $\alpha=0$ & $x_{0}=0.1$ \\
\hline
\end{tabular}




\begin{tabular}{|l|l|l|l|}
\hline$f_{2}(x)=\left(x^{2}-1\right)^{-1}-1$ & $m=0$ & $\alpha=1.4142 \ldots$ & $x_{0}=1.25$ \\
\hline$f_{3}(x)=(x-2)\left(x^{10}+x+1\right) \exp (-x-1)$ & $m=-2$ & $\alpha=2$ & $x_{0}=0.25$ \\
\hline$f_{4}(x)=(x+1) \exp (\sin (x))-x^{2} \exp (\cos (x))-1$ & $m=12$ & $\alpha=0$ & $x_{0}=1.3$ \\
\hline$f_{5}(x)=\sin (x)^{2}-x^{2}+1$ & $m=12$ & $\alpha=1.4044 \ldots$ & $x_{0}=1$ \\
\hline$f_{6}(x)=\exp (1-x)-\cos \left(2^{-1} x\right)$ & $m=-6$ & $\alpha=1.1878 \ldots$ & $x_{0}=3$ \\
\hline$f_{7}(x)=\ln \left(x^{2}+x+2\right)-x+1$ & $m=15$ & $\alpha=4.1525 \ldots$ & $x_{0}=0.75$ \\
\hline$f_{8}(x)=6 x^{10}-2 x^{3}-x+2$ & $m=-32$ & $\alpha=0.8954 \ldots$ & $x_{0}=0.5$ \\
\hline$f_{9}(x)=\cos (x)^{2}-2^{-1} x$ & $m=33$ & $\alpha=0.8570 \ldots$ & $x_{0}=1.4$ \\
\hline$f_{10}(x)=x^{15}+x^{4}-4 x^{2}+25$ & $m=-2$ & $\alpha=-1.2259 \ldots$ & $x_{0}=-1.4$ \\
\hline
\end{tabular}

Table 2 Errors occurring in the estimates of the simple root of (40) by the methods described

\begin{tabular}{lccccc}
\hline$f_{i}$ & $\mathbf{( 5 )}$ & $\mathbf{( 9 )}: \mathbf{i}=\mathbf{1}$ & $\mathbf{( 9 ) : i = 2}$ & $\mathbf{( 9 )}: \mathbf{i}=\mathbf{3}$ & $\mathbf{( 9 ) : i = 4}$ \\
\hline$f_{1}$ & $0.114 \mathrm{e}-62$ & $0.366 \mathrm{e}-157$ & $0.119 \mathrm{e}-158$ & $0.213 \mathrm{e}-158$ & $0.320 \mathrm{e}-186$ \\
\hline$f_{2}$ & $0.445 \mathrm{e}-32$ & $0.270 \mathrm{e}-65$ & $0.148 \mathrm{e}-79$ & $0.272 \mathrm{e}-76$ & $0.569 \mathrm{e}-94$ \\
\hline$f_{3}$ & $0.203 \mathrm{e}-40$ & $0.928 \mathrm{e}-91$ & $0.387 \mathrm{e}-98$ & $0.100 \mathrm{e}-96$ & $0.981 \mathrm{e}-122$ \\
\hline$f_{4}$ & $0.895 \mathrm{e}-69$ & $0.173 \mathrm{e}-135$ & $0.106 \mathrm{e}-231$ & $0.104 \mathrm{e}-188$ & $0.435 \mathrm{e}-244$ \\
\hline$f_{5}$ & $0.342 \mathrm{e}-85$ & $0.278 \mathrm{e}-209$ & $0.108 \mathrm{e}-214$ & $0.967 \mathrm{e}-214$ & $0.860 \mathrm{e}-331$ \\
\hline$f_{6}$ & $0.112 \mathrm{e}-64$ & $0.125 \mathrm{e}-160$ & $0.106 \mathrm{e}-167$ & $0.213 \mathrm{e}-166$ & $0.147 \mathrm{e}-277$ \\
\hline$f_{7}$ & $0.968 \mathrm{e}-85$ & $0.804 \mathrm{e}-247$ & $0.893 \mathrm{e}-191$ & $0.279 \mathrm{e}-196$ & $0.492 \mathrm{e}-248$ \\
\hline$f_{8}$ & $0.275 \mathrm{e}-41$ & $0.790 \mathrm{e}-108$ & $0.152 \mathrm{e}-85$ & $0.152 \mathrm{e}-89$ & $0.166 \mathrm{e}-111$ \\
\hline$f_{9}$ & $0.981 \mathrm{e}-114$ & $0.562 \mathrm{e}-233$ & $0.292 \mathrm{e}-179$ & $0.271 \mathrm{e}-195$ & $0.821 \mathrm{e}-318$ \\
\hline$f_{10}$ & $0.162 \mathrm{e}-15$ & $0.717 \mathrm{e}-28$ & $0.580 \mathrm{e}-33$ & $0.649 \mathrm{e}-32$ & $0.115 \mathrm{e}-75$ \\
\hline & & & & & \\
\hline
\end{tabular}

Table 3 Performance of COC 


\begin{tabular}{|c|c|c|c|c|c|}
\hline$f_{i}$ & (5) & (9): $i=1$ & (9):i=2 & (9): $i=3$ & (9): $i=4$ \\
\hline$f_{1}$ & 3.0000 & 4.0000 & 4.0000 & 4.0000 & 4.0000 \\
\hline$f_{2}$ & 3.0001 & 4.0000 & 4.0000 & 4.0000 & 4.0000 \\
\hline$f_{3}$ & 3.0000 & 4.0000 & 4.0000 & 4.0000 & 4.0000 \\
\hline$f_{4}$ & 3.0000 & 4.0000 & 4.0000 & 4.0000 & 4.0000 \\
\hline$f_{5}$ & 3.0000 & 4.0000 & 4.0000 & 4.0000 & 4.0000 \\
\hline$f_{6}$ & 3.0000 & 4.0000 & 4.0000 & 4.0000 & 4.0000 \\
\hline$f_{7}$ & 3.0000 & 4.0000 & 4.0000 & 4.0000 & 4.0000 \\
\hline$f_{8}$ & 3.0000 & 4.0000 & 4.0000 & 4.0000 & 3.9828 \\
\hline$f_{9}$ & 3.0000 & 4.0000 & 4.0000 & 4.0000 & 4.0000 \\
\hline$f_{10}$ & 3.0052 & 4.0000 & 4.0000 & 4.0000 & 4.0000 \\
\hline
\end{tabular}

\section{Conclusion}

In this paper, we have demonstrated the performance of the new iterative method, namely the Simpson-type fourth-order iterative method. The prime motive of the development of the new fourth-order method was to establish a higher order of convergence method than the classical Simpson third-order iterative method. Furthermore, we have introduced a family for approximating the second-order derivative. Numerical comparisons are made to show the performance of the derived method. We have examined the effectiveness of the new fourth-order iterative method by showing the accuracy of the simple root of a nonlinear equation. In addition, it should be noted that the new formula for approximating second-order derivative in the new method (9) is dependent on the value of $m$, therefore a particular choice $m$ is very important. Hence further study is needed to establish the optimal estimate of simple root.

\section{Competing Interests}

The author declares that no competing interests exist.

\section{References}

[1] N. Ahmad, V. P. Singh, A New Iterative Method for Solving Nonlinear Equations Using Simpson Method, Inter. J. Math. Appl. 5 (4) (2017) 189-193.

[2] Eskandari, H. (2017) Simpson's Method for Solution of Nonlinear Equation. Appl. Math. 8 (2017) 929933.

[3] W. Gautschi, Numerical Analysis: An Introduction, Birkhauser, 1997. 
[4] V. I. Hasanov, I. G. Ivanov, G. Nedjibov, A New modification of Newton's method, Appl. Math. Eng (2015) 278-286.

[5] J. Jayakumar, Generalized Simpson-Newton's Method for Solving Nonlinear Equations with Cubic Convergence, J. Math. 7 (5) (2013) 58-61

[6] G. Nedjibov, V. I. Hasanov, M. G. Petkov, on some families of multi-point iterative methods for solving nonlinear equations, Numer. Algo. 42 (2) (2006) 127-136.

[7] A. M. Ostrowski, Solutions of equations and system of equations, Academic Press, New York, 1960.

[8] M. S. Petkovic, B. Neta, L. D. Petkovic, J. Dzunic, Multipoint methods for solving nonlinear equations, Elsevier 2012.

[9] R. Thukral, New modifications of Newton-type methods with eighth-order convergence for solving nonlinear equations, J. Adv. Math. Vol 10 (3) (2015) 3362-3373.

[10] J. F. Traub, Iterative Methods for solution of equations, Chelsea publishing company, New York 1977. 